\title{
EL EMPLEO DE LA RED SOCIAL TIKTOK POR LOS EQUIPOS DE FÚTBOL DE PRIMERA DIVISIÓN DE LA LIGA ESPAÑOLA
}

\section{THE USE OF THE SOCIAL NETWORK TIKTOK BY THE FIRST DIVISION SOCCER TEAMS OF THE SPANISH LEAGUE}

\author{
Pavel Sidorenko Bautista \\ Universidad Francisco de Vitoria, España \\ (iD) https://orcid.org/0000-0002-8094-3089 \\ Nadia Alonso-López \\ Universidad Politécnica de Valencia, España \\ (iD) https://orcid.org/0000-0002-5220-2232

\section{Raúl Terol-Bolinches} \\ Universidad Politécnica de Valencia, España \\ (iD) https://orcid.org/0000-0002-0915-7288
}

Autor para correspondencia: Pavel Sidorenko Bautista, email: pavel.sidorenko@ufv.es

\section{Resumen}

Los avances tecnológicos, la hiperconectividad y la proliferación de dispositivos inteligentes son tres rasgos que caracterizan el actual entorno mediático. En este contexto, el $62 \%$ de las personas utilizan las redes sociales con una media de uso de dos horas diarias. Entre ellas, TikTok es una de las que más ha crecido en usuarios el último año, situándose como la sexta red social más utilizada en el mundo a finales de 2020 según Hootsuit. TikTok es una plataforma de uso mayoritariamente por parte del público joven, que se siente atraído principalmente por tres características: el vínculo emocional con el contenido, la interacción y la usabilidad. Asimismo, el consumo de vídeos cortos está en alza y el contenido en formato vertical, corto, creativo y adaptado al consumo de móviles predomina en todos los sentidos. La presente investigación se plantea en un ámbito escasamente estudiado como es el uso de esta plataforma en el deporte, concretamente, por parte de los clubes de fútbol que compiten en la Primera División española, fijando así el objetivo principal. Como objetivos secundarios se plantea estudiar la tipología de publicaciones de los clubes y establecer el contenido y narrativas que generan más engagement con los seguidores.

Palabras clave: TikTok, fútbol, Liga Santander, redes sociales, comunicación organizacional.

Global Media Journal México, 18(35), 32-54, julio - diciembre 2021. 


\begin{abstract}
Technological advances, hyperconnectivity and the proliferation of smart devices are three features that characterize the current media environment. In this context, $62 \%$ of people use social networks for an average of two hours a day. Among them, TikTok is one of those that has grown the most in users in the last year, ranking as the sixth most used social network in the world. TikTok is a platform mostly used by a young audience, which is attracted mainly by the emotional bond with the content, interaction, and usability. Also, the consumption of short videos is on the rise and content in vertical format, short, creative, and adapted to mobile consumption predominates in every way. The present research arises in a scarcely studied area such as the use of this platform in the field of sport, specifically, by soccer clubs competing in the Spanish First Division, thus setting the main objective. The secondary objectives are to study the type of content published by the clubs and to establish the content and narratives that generate the most engagement with followers. The methodology used is content analysis with quantitative indicators such as the number of publications of each team, followers and 'likes'.
\end{abstract}

Keywords: TikTok, football, Liga Santander, social media, organizational communication.

Recibido: 08/07/2021

Aceptado: 14/10/2021

\section{Introducción}

El fútbol es, de entre todos los deportes, el que logra captar mejor la atención de los medios de comunicación por el enorme interés que suscita entre la ciudadanía (Meneses \& Ávalos, 2013), convirtiéndose en la base del negocio sobre el que se edifican las empresas periodísticas (Rojas-Torrijos, 2012). Su relevancia y repercusión en la sociedad ha obligado a los clubes de fútbol a mantener una presencia firme en el ecosistema comunicativo, es por ello que la gestión de la comunicación se debe desenvolver desde la propia organización, debiendo ser capaces de gestionar una estrategia comunicativa que posibilite la planificación, el desarrollo, la ejecución y la evaluación de la comunicación realizada (Castillo-Esparcia et al., 2016). De esta manera, los equipos de fútbol más importantes se han transformado en "multinacionales del entretenimiento" (Ginesta, 2011; Soriano, 2009) mediante un proceso de cambio de su modelo empresarial que tiene por objetivos aumentar los beneficios económicos $\mathrm{y}$ fortalecer su posicionamiento comunicativo en la red (Tejedor et al., 2020).

Las tecnologías de la comunicación han hecho posible que se produzca un cambio sustancial en la manera de coordinar la comunicación y las relaciones públicas en las organizaciones, aportando mejoras y generando nuevas oportunidades y ventajas (Almansa \& Castillo-Esparcia, 2014). Estas 
tecnologías, además, han fomentado que los clubes se hayan convertido en canales comunicativos (Tejedor et al., 2020), creando medios de comunicación propios en muchos de los clubes, como canales de televisión o emisoras de radio. Todo ello ha llevado consigo una reinvención por parte de los clubes de fútbol (López \& Fernández, 2015) y ha demostrado la capacidad de adaptación a los nuevos medios y redes sociales que han surgido y surgen en la red, como se verá en el caso de estudio del presente artículo, así como a las nuevas narrativas que llevan aparejadas (Cano, 2017).

\section{Marco teórico}

\section{Sociedad de la información, ciudadanía digital, redes sociales y clubes de fútbol}

La sociedad de la información se ha construido a lo largo de las últimas décadas sobre un sistema de redes y herramientas tecnológicas, pero en el que las personas desempeñan un papel fundamental en este escenario (Terol-Bolinches, 2016). Las redes sociales, que tan populares se han convertido y a las que cada vez más se puede acceder con mayor facilidad, han creado una especie de ecosistema de subculturas y grupos que se han ido estableciendo de manera espontánea, por este motivo, Rheingold (2002) defiende que las repercusiones sociales que conlleva la convergencia de las tecnologías resultan verdaderamente significativas a nivel social, adoptando las personas nuevas formas de interactuar, coordinar o cooperar.

Las audiencias digitales potencian la condición libre, activa, participativa, crítica y responsable en el nuevo espacio creado por la sociedad de la información (Gozàlvez, 2012). De esta manera, en tanto se comparten las redes sociales, la confianza o los valores que promueven la colaboración y cooperación entre las personas, se genera capital social y el intercambio de conocimiento colectivo, favorecido, como ya se ha destacado, por las tecnologías que lo posibilitan. (Cobo \& Pardo, 2007).

La web 2.0 convirtió a la red de un simple escaparate digital a una plataforma abierta, en la que la arquitectura de la participación de sus usuarios iba a ser una de las características principales y uno de los términos que retroalimentarían la evolución de esta. Otro de los conceptos sería el de las redes sociales, que se desarrolló con la llegada de la web 2.0 y que se ha ido consolidando a lo largo de los últimos lustros (Cobo \& Pardo, 2007). La aparición del flujo multidireccional de los mensajes nos lleva al concepto de prosumidores, de ahí la importancia de las personas en este escenario digital, ahora los usuarios se convierten a la par en consumidores y productores de información y contenidos (Sotelo, 2012). Castillo-Esparcia et al. (2016) destacan que los aspectos que caracterizan a las formas de comunicación que se han implementado en esta sociedad de la información son la incertidumbre, la simetría, la complejidad, la viralidad, la implicación y la multimedialidad, que conforman una nueva forma de instaurar relaciones satisfactorias y que 
tengan un interés común con los públicos de los clubes de fútbol como instituciones.

Las redes sociales han servido de gran potencial al ámbito del fútbol, por lo que se refiere a la difusión sus mensajes y a la promoción (García del Barrio, 2016). Aunque, concretamente en este ámbito, la gestión que de ellas se ha realizado por parte de los clubes ha sido fundamentalmente unidireccional (Castillo-Esparcia et al., 2016). Si las páginas web son un canal que ofrece información en una única dirección (noticias del club, comunicados, productos), la presencia de los clubes en las redes sociales no dista demasiado, siendo también utilizadas para propagar sus comunicados, en lugar de aprovecharlas para interactuar con sus seguidores y aficionados (Sotelo, 2012).

El contexto de la COVID-19 con sus consecuentes confinamientos sociales radicales generó una distorsión importante en los calendarios $\mathrm{y}$ actividades regulares de competiciones y organizaciones deportivas de todo el mundo, al tiempo que obligó a equipos y atletas a estrechar su relación con su público y seguidores. Así, los canales digitales resultaron la única vía para mantener el contacto y optar por la posibilidad de incrementar el alcance, obligando así a innovar en los canales y en la narrativa (Ruihley \& Li, 2020).

Si ya existía una tendencia por construir lovemarks en torno a los equipos explotando las redes sociales como principales canales (Miranda Peña, 2021), el contexto sanitario antes descrito aceleró este proceso sobre todo de cara a nuevas plataformas $\mathrm{y}$ audiencias.

\section{La red social TikTok}

Se trata de una plataforma cuyo origen remite a su antecesora Music.ly, que hasta 2016 se enfocaba en playbacks y coreografías realizadas por audiencias muy jóvenes.

Ante el éxito de otras redes sociales como Snapchat y Dubsmash en el seno de la generación Z, la china ByteDance decidió evolucionar esta aplicación en lo que hoy se conoce como TikTok: un entorno digital que hibrida la propuesta de estas tres ya mencionadas donde impera la narrativa vertical en código de entretenimiento, mediante grandes cantidades de estímulo visual y exigencia creativa, procurando dinamismo tanto para producir como para consumir los contenidos (Li et al., 2019; Shuai et al., 2019).

En otras palabras, TikTok, mantiene su propuesta de comunicación con base en playbacks, así como por propuestas divertidas y que instan al relajamiento y entretenimiento de su comunidad de usuarios (Yu-Liang et al., 2019), permitiendo el desarrollo de nuevas propuestas en torno al concepto de comunicación efímera como el caso de los microtutoriales en 60 segundos.

El hecho de que haya superado los 800 millones de usuarios a nivel global en 2020 le permitió ostentar el logro de ser la app móvil más descargada de dicho año en todo el mundo (Ditrendia, 2020), récord que ha mantenido hasta el primer cuatrimestre de 2021 (Chan, 2021). Y aunque inicialmente sus usuarios fueran mayoritariamente de la generación Z (Li et al., 2019; Rapkin, 2017; Shuai et al., 2019), el contexto de la pandemia por COVID-

Global Media Journal México, 18(35), 32-54, julio - diciembre 2021. 
19 y los consecuentes confinamientos radicales, ocasionaron un importante incremento de usuarios millennials e inclusive de la generación $\mathrm{X}^{\mathrm{i}}$.

Asimismo, en la actualidad, la importancia de esta red social está determinada por el hecho de haber sido seleccionada en el ranking de las 100 empresas más valiosas del mundo por parte de la plataforma de valor de marca Brand Z (Kantar, 2020).

Se trata de una tendencia digital mundial que ha ocasionado que emisores de muy diversa índole se hayan sumado a ella, innovando y adaptando sus mensajes a un código y una audiencia que a veces resultan ajenos a su actividad o propuesta (Sidorenko et al., 2020; Vásquez et al., 2020).

Destaca de este proceso una narrativa más natural, informal e inclusive en modo "tras cámaras". Se trata de una propuesta en código de antimarketing en su acepción positiva (Sidorenko et al., 2021), mediante el cual los creadores de contenidos intentan mostrarse tal y como son, reafirmando así la confianza de la audiencia con respecto a ellos (Permana \& Meinarni, 2021). Es una red social que estimula la creatividad y el pensamiento crítico de la comunidad de usuarios sobre la premisa de la identidad digital (Omar \& Dequan, 2020; Van den Bremer \& Siebelink, 2020).

El algoritmo que opera la plataforma es una inteligencia artificial que procura la exhibición de los contenidos de la comunidad de usuarios a través de un feed principal, con base en variables como: tiempo de visualización que le dedican los usuarios, comentarios, likes, las veces que es compartido a través de otras vías digitales, si se utiliza un audio de fondo y qué tipo de audio es, y los hashtags.

Particularmente los audios constituyen un formato aparte dentro de la plataforma, y están concebidos para darle mayor contexto a la propuesta audiovisual. De acuerdo con la experiencia de usuario y observaciones realizadas para el presente trabajo, es posible sentenciar que si un audio es tendencia porque muchos usuarios lo utilizan como fondo para sus creaciones, el algoritmo tomará en consideración cualquier publicación que lo ejecute para permitir que esa tendencia prevalezca un tiempo mayor en exposición, y así asegurar una mayor implicación por parte de esa comunidad de usuarios.

\section{Metodología}

La red social TikTok se erigió como un fenómeno digital global durante el 2020, como consecuencia principalmente de los primeros confinamientos radicales por la COVID-19 (Sidorenko et al., 2020), llegando a ser la sexta red social más utilizada a escala global (Sehl, 2020) y la sexta red social en frecuencia de uso en España hasta el primer cuatrimestre de 2021 (IAB, 2021).

Ante este escenario, han sido muchos los creadores de contenidos y emisores que han buscado adaptarse rápidamente a esta plataforma con el fin de incrementar el alcance de su mensaje de cara a nuevos segmentos de audiencia.

A través de esta investigación se propone someter a estudio cómo comunican los clubes de fútbol español 
integrantes de La Liga Santander (primera división) en la temporada 2020/2021 en la red social TikTok, planteándose las siguientes preguntas de investigación: ¿sobre qué temas hacen mayor énfasis?, ¿qué tanto les ha condicionado el particular código del mensaje de esta plataforma? y iles ha costado desarrollar una comunicación diferenciada con respecto a las otros canales digitales que utilizan?

Las hipótesis de partida son:

H1. Al ser tendencia digital, los clubes de fútbol de La Liga Santander han sumado TikTok como nuevo canal de comunicación, en especial desde el segundo trimestre de 2020.

H2. El contenido de los clubes de fútbol de La Liga Santander en TikTok está construido mayoritariamente sobre una narrativa en clave publicitaria o de carácter anecdótico con especial énfasis en la imagen del club.

H3. De momento, TikTok no está siendo empleada por los clubes de fútbol de La Liga Santander como una vía para la comunicación corporativa.

H4. El desarrollo de contenidos en TikTok por parte de los clubes de fútbol de La Liga Santander es aún incipiente y experimental, con una escasa producción multimedia.

Para tal fin se ha realizado una revisión a través del buscador interno de la red social para evidenciar cuáles equipos de la primera división española de fútbol (temporada 20/21) tenían perfiles activos. De esta forma, se ha obtenido como evidencia que, de los 20 clubes integrantes de dicha competición, 19 tenían presencia en TikTok: Athletic de Bilbao, Atlético de Madrid, Barcelona, Cádiz C.F., Celta,
Deportivo Alavés, Eibar, Elche, Getafe, Granada C.F., Levante, Osasuna, Real Sociedad, Real Madrid, Real Valladolid, Sevilla F.C., SD Huesca, Valencia y Villarreal.

En el caso particular del Real Betis Balompié (perfil verificado) hasta el 31 de diciembre de 2020 no tenía ningún contenido publicado y por tanto ha quedado fuera del estudio. Cabe destacar que observaciones posteriores permiten afirmar que hasta el primer cuatrimestre de 2021 no han comenzado actividades de manera orgánica a través de la plataforma.

El estudio tiene un enfoque cuantitativo y cualitativo. La muestra ha constado de 2169 contenidos en total desde el inicio de actividades en TikTok por parte de cada club hasta el 31 de diciembre de 2020 y los datos han sido consolidados en una tabla de análisis donde por perfil se ha procedido a la cuantificación de contenidos por tono o intención narrativa y categoría.

En atención al código del mensaje impuesto por la comunidad de usuarios en TikTok, así como por el interés de comunicación sobre tópicos específicos por parte de los clubes de fútbol, se determinaron como categorías de contenido:

- Hashtag-challenges (reto-etiquetas)

- Reposición de jugadas

- Entrenamientos

- Jugadores protagónicos pre, durante y post partido

- Aficiones protagonistas pre, durante y post partido

- Enfoque en solo jugador

- Celebraciones 
- Eventos/Competiciones

- Alusión al equipo rival

- Identidad del Club

Se entiende por Hashtag-challenges las iniciativas que promueven la implicación de los usuarios a partir de un contenido, imitándolo y haciendo nuevas proposiciones a partir de ello. Por su parte, al hacer referencia a "jugadores protagónicos pre, durante y post partido" se hace con la intención de señalar contenidos con especial enfoque en el equipo en diversas situaciones que no correspondan a entrenamientos o reposiciones de jugadas (cenas, convivencias, concentraciones, actividades benéficas, etc.). Mismo caso que con "Aficiones protagonistas pre, durante y post partido", que elude a la eventual visibilidad que le da el club a su hinchada a través de esta plataforma.

Asimismo, se ha determinado cruzar estas categorías con la intención o carácter narrativo específico que le han querido asignar o que se interpreta en las publicaciones estudiadas:

- Informativo (actualidad del club: entrenamientos, traslados, concentraciones, etc.)

- Publicitario (mercancía alusiva a la institución, eventos especiales, colaboraciones con marcas, etc.)
- Entretenido (challenges, performance, bromas, contenido extradeportivo de carácter memético)

- Anecdótico (referencias a hazañas pasadas del club o de jugadores específicos)

- Comunicación institucional (economía, responsabilidad social, desempeño deportivo, etc.)

En resumen, el presente estudio tiene como objetivo determinar de qué manera están comunicando a través de TikTok los clubes de fútbol de la primera división española, y si se han adaptado efectivamente a las particularidades narrativas de esta red social que ratifica su condición de tendencia digital global.

\section{Resultados}

De la revisión planteada se han obtenido 2169 publicaciones en total desde que cada club de La Liga Santander (temporada 20/21) comenzara su actividad en TikTok hasta el 31 de diciembre de 2020.

De esta muestra (Tabla 1) se desprende a primera vista que hay una producción de contenidos desigual por parte de cada club, pero también un esfuerzo por incorporarse a lo que es hoy una red social tendencia a escala global. 


\section{Tabla 1}

Comunicación de los equipos de la Liga Santander con presencia en TikTok desde el inicio del perfil hasta el 31 de diciembre 2020

\section{Tipo y tono del contenido}

\begin{tabular}{|c|c|c|c|c|c|c|}
\hline Club & & & & 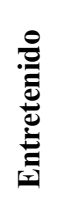 & $\frac{8}{\stackrel{\circlearrowright}{0}}$ & 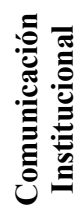 \\
\hline \multirow{10}{*}{$\begin{array}{c}\text { Athletic de Bilbao } \\
\text { @athleticclub } \\
\text { (perfil verificado) } \\
02 / 05 / 2020\end{array}$} & Hahstag-Challenge (retos) y/o bromas & 1 & & 3 & & \\
\hline & Reposición de jugadas & 1 & & 6 & & \\
\hline & Entrenamientos & 4 & & 9 & & \\
\hline & Jugadores protagonistas pre y post partido & 2 & & 7 & & \\
\hline & Aficionados protagonistas pre, durante y post partido & & & 1 & & \\
\hline & Enfoque en un solo jugador & 1 & 4 & 7 & & \\
\hline & Celebraciones & & & & & \\
\hline & Eventos/Competiciones & & 2 & 2 & & \\
\hline & Alusión a equipo rival & & & & & \\
\hline & Identidad del club & 2 & 7 & 12 & 2 & 3 \\
\hline \multirow{9}{*}{$\begin{array}{c}\text { Atlético de Madrid } \\
\text { @atleticodemadrid } \\
\text { (perfil verificado) } \\
27 / 06 / 2019\end{array}$} & $\begin{array}{l}\text { Hahstag-Challenge (retos) y/o bromas } \\
\text { Reposición de jugadas }\end{array}$ & 2 & & & & \\
\hline & Entrenamientos & 219 & & 4 & & 4 \\
\hline & Jugadores protagonistas pre y post partido & 64 & & 1 & & \\
\hline & Aficionados protagonistas pre, durante y post partido & 36 & 2 & & & 12 \\
\hline & Enfoque en un solo jugador & 83 & 3 & 5 & 3 & 4 \\
\hline & Celebraciones & & & & & 1 \\
\hline & Eventos/Competiciones & 39 & 3 & 7 & 1 & 29 \\
\hline & Alusión a equipo rival & 1 & & & & \\
\hline & Identidad del club & 5 & 22 & 3 & 1 & 32 \\
\hline \multirow{10}{*}{$\begin{array}{c}\text { FC Barcelona } \\
@ \text { fcbarcelona } \\
\text { (perfil verificado) } \\
05 / 07 / 2019\end{array}$} & Hahstag-Challenge (retos) y/o bromas & & & 51 & 18 & 2 \\
\hline & Reposición de jugadas & 14 & & 5 & & \\
\hline & Entrenamientos & 5 & & 34 & 15 & \\
\hline & Jugadores protagonistas pre y post partido & 7 & & 23 & 9 & 2 \\
\hline & Aficionados protagonistas pre, durante y post partido & 1 & & 2 & 3 & \\
\hline & Enfoque en un solo jugador & 10 & 2 & 55 & 35 & 20 \\
\hline & Celebraciones & & & 1 & & \\
\hline & Eventos/Competiciones & & & 3 & 1 & 1 \\
\hline & Alusión a equipo rival & 3 & & 1 & 2 & \\
\hline & Identidad del club & 2 & 4 & 12 & 11 & 13 \\
\hline \multirow{10}{*}{$\begin{array}{c}\text { Cádiz CF } \\
\text { @cadizclubdefutbol } \\
\text { (perfil verificado) } \\
17 / 06 / 2019\end{array}$} & Hahstag-Challenge (retos) y/o bromas & 1 & & 9 & 1 & \\
\hline & Reposición de jugadas & 71 & & 18 & & \\
\hline & Entrenamientos & 23 & & 25 & 1 & \\
\hline & Jugadores protagonistas pre y post partido & 4 & & & & \\
\hline & Aficionados protagonistas pre, durante y post partido & 2 & & & & \\
\hline & Enfoque en un solo jugador & 2 & & 22 & 8 & \\
\hline & Celebraciones & 4 & & 1 & & \\
\hline & Eventos/Competiciones & & & & & \\
\hline & Alusión a equipo rival & 1 & & & & \\
\hline & Identidad del club & 10 & 3 & 20 & 6 & 4 \\
\hline
\end{tabular}




\section{Tipo y tono del contenido}

\begin{tabular}{|c|c|c|c|c|c|c|}
\hline Club & & 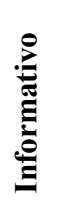 & ڤํㅡㄹ & 胥 & 递 & 己 \\
\hline $\begin{array}{c}\text { Celta } \\
\text { @rccelta } \\
\text { (perfil verificado) } \\
24 / 07 / 2020\end{array}$ & $\begin{array}{l}\text { Hahstag-Challenge (retos) y/o bromas } \\
\text { Reposición de jugadas } \\
\text { Entrenamientos } \\
\text { Jugadores protagonistas pre y post partido } \\
\text { Aficionados protagonistas pre, durante y post partido } \\
\text { Enfoque en un solo jugador } \\
\text { Celebraciones } \\
\text { Eventos/Competiciones } \\
\text { Alusión a equipo rival } \\
\text { Identidad del club }\end{array}$ & 1 & 1 & $\begin{array}{c}12 \\
8 \\
2\end{array}$ & 4 & 1 \\
\hline $\begin{array}{c}\text { Deportivo Alavés } \\
\text { @deportivoalaves } \\
\text { (perfil verificado) } \\
12 / 11 / 2020\end{array}$ & $\begin{array}{l}\text { Hahstag-Challenge (retos) y/o bromas } \\
\text { Reposición de jugadas } \\
\text { Entrenamientos } \\
\text { Jugadores protagonistas pre y post partido } \\
\text { Aficionados protagonistas pre, durante y post partido } \\
\text { Enfoque en un solo jugador } \\
\text { Celebraciones } \\
\text { Eventos/Competiciones } \\
\text { Alusión a equipo rival } \\
\text { Identidad del club }\end{array}$ & 1 & & $\begin{array}{c}11 \\
4 \\
1\end{array}$ & 1 & \\
\hline $\begin{array}{c}\text { Eibar } \\
\text { @sdeibar } \\
\text { (perfil verificado) } \\
19 / 12 / 2019\end{array}$ & $\begin{array}{l}\text { Hahstag-Challenge (retos) y/o bromas } \\
\text { Reposición de jugadas } \\
\text { Entrenamientos } \\
\text { Jugadores protagonistas pre y post partido } \\
\text { Aficionados protagonistas pre, durante y post partido } \\
\text { Enfoque en un solo jugador } \\
\text { Celebraciones } \\
\text { Eventos/Competiciones } \\
\text { Alusión a equipo rival } \\
\text { Identidad del club }\end{array}$ & $\begin{array}{l}1 \\
3 \\
3\end{array}$ & 4 & $\begin{array}{c}12 \\
4\end{array}$ & 1 & \\
\hline $\begin{array}{c}\text { Elche CF } \\
@ \text { elchecf } \\
\text { (perfil verificado) } \\
08 / 05 / 2020\end{array}$ & $\begin{array}{l}\text { Hahstag-Challenge (retos) y/o bromas } \\
\text { Reposición de jugadas } \\
\text { Entrenamientos } \\
\text { Jugadores protagonistas pre y post partido } \\
\text { Aficionados protagonistas pre, durante y post partido } \\
\text { Enfoque en un solo jugador } \\
\text { Celebraciones } \\
\text { Eventos/Competiciones } \\
\text { Alusión a equipo rival } \\
\text { Identidad del club }\end{array}$ & 1 & & $\begin{array}{l}5 \\
2 \\
5 \\
1 \\
1 \\
7\end{array}$ & 1 & \\
\hline $\begin{array}{c}\text { Getafe CF } \\
@ \text { getafecf } \\
\text { (perfil verificado) } \\
09 / 05 / 2020\end{array}$ & $\begin{array}{l}\text { Hahstag-Challenge (retos) y/o bromas } \\
\text { Reposición de jugadas } \\
\text { Entrenamientos } \\
\text { Jugadores protagonistas pre y post partido } \\
\text { Aficionados protagonistas pre, durante y post partido } \\
\text { Enfoque en un solo jugador } \\
\text { Celebraciones } \\
\text { Eventos/Competiciones } \\
\text { Alusión a equipo rival } \\
\text { Identidad del club }\end{array}$ & $\begin{array}{c}15 \\
2 \\
3 \\
1\end{array}$ & 1 & 2 & 1 & 2 \\
\hline
\end{tabular}

Global Media Journal México, 18(35), 32-54, julio - diciembre 2021. 


\section{Tipo y tono del contenido}

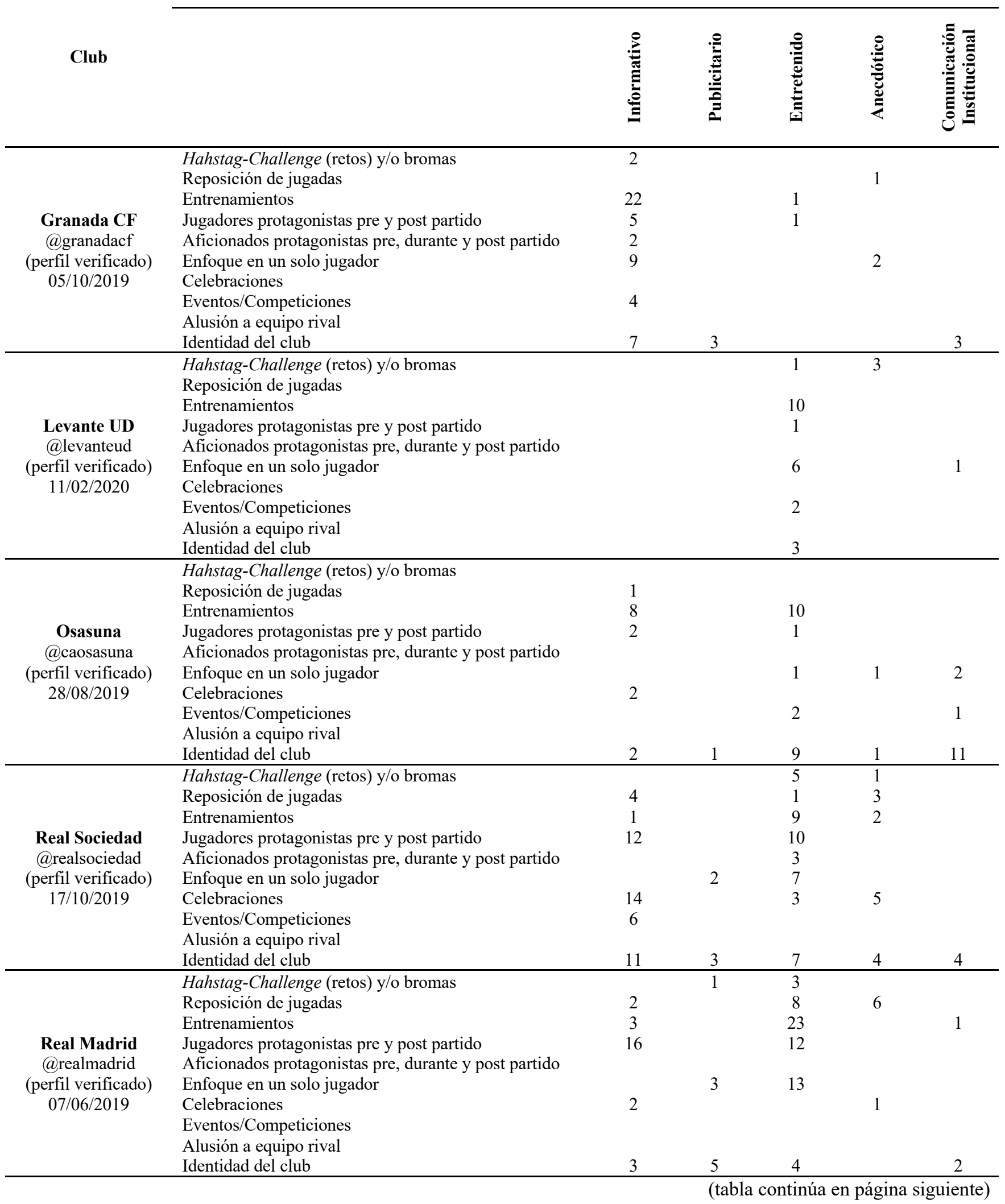

Global Media Journal México, 18(35), 32-54, julio - diciembre 2021. 
En primera instancia es necesario precisar que la incursión de este tipo de emisores en TikTok se ha venido realizando desde el segundo trimestre de 2019 hasta el último de 2020, reportándose el pico más alto de nuevas altas en el segundo trimestre de 2020 (Figura 1) coincidiendo con los primeros confinamientos radicales por la COVID-19 y la consecuente 'viralización' de la red social.

\section{Figura 1}

Trimestre en el que iniciaron actividades en TikTok los clubes de fútbol de La Liga Santander

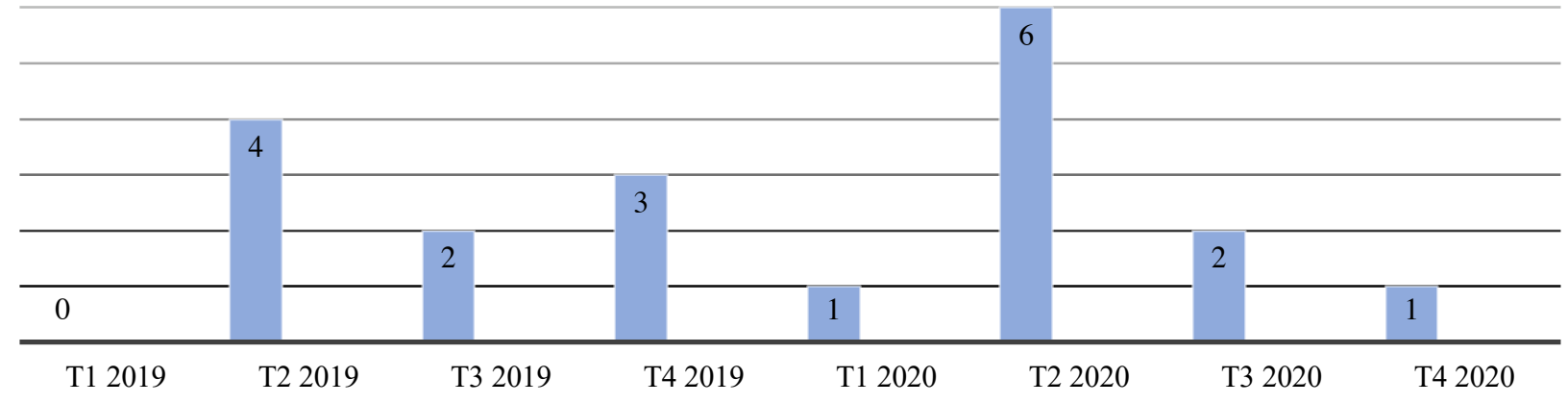

\section{Figura 2}

Tipología de contenidos más utilizados por los equipos de la Liga Santander en TikTok desde su creación hasta el 31 de diciembre 2020
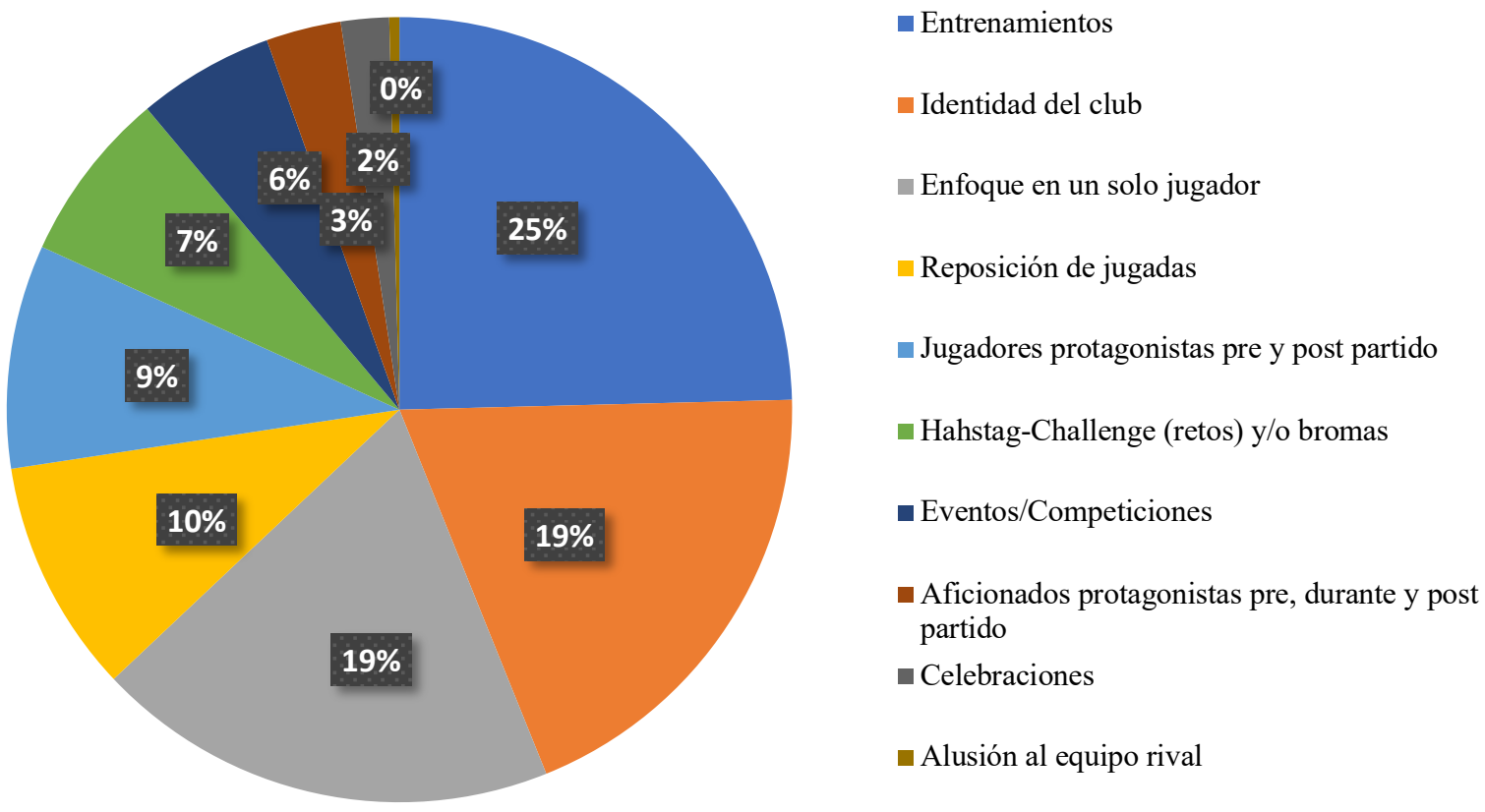

- Alusión al equipo rival 
Según los datos de la Tabla 1 y la Figura 2, la comunicación de los clubes de la máxima categoría del fútbol español en TikTok está enfocada principalmente en los entrenamientos (25\%), identidad del club (19\%) o en un jugador en particular (19\%). Queda en evidencia en menor proporción publicaciones que aludan a reposiciones de jugadas, actividades de los jugadores al margen de partidos o entrenamientos y el resto de las categorías ya establecidas en ambos soportes de medición.

Determinando el carácter narrativo de este tipo de comunicación, según se muestra en la Tabla 2, cuando los clubes crean contenidos relacionados con los entrenamientos, lo hacen mayoritariamente con carácter informativo. Si aluden a la identidad del club, la actividad denota un tono publicitario.

\section{Tabla 2}

Comparativa entre tono y tipología de contenidos más utilizados por los equipos de la Liga Santander en TikTok desde su creación hasta el 31 de diciembre 2020

\begin{tabular}{lccccc}
\hline & Informativo & Publicitario & Entretenido & Anecdótico & $\begin{array}{c}\text { Comunicación } \\
\text { Institucional }\end{array}$ \\
\hline Hahstag-Challenge (retos) & 6 & 1 & 106 & 37 & 4 \\
y/o bromas & 107 & 0 & 82 & 19 & 0 \\
Reposición de jugadas & 301 & 0 & 205 & 23 & 5 \\
Entrenamientos & 115 & 0 & 74 & 9 & 2 \\
Jugadores protagonistas pre & & 2 & 7 & 6 & 12 \\
y post partido & 41 & 18 & 164 & 91 & 27 \\
Aficionados protagonistas & 112 & 0 & 7 & 10 & 1 \\
pre, durante y post partido & 25 & 5 & 27 & 5 & 31 \\
Enfoque en un solo jugador & 54 & 0 & 2 & 2 & 0 \\
Celebraciones & 5 & 72 & 150 & 66 & 81 \\
Eventos/Competiciones & 50 & 98 & 824 & 268 & 163 \\
Alusión a equipo rival & 816 & & & & \\
Identidad del club & & & & \\
$\quad$ Totales & & & & & \\
\end{tabular}

Los contenidos entretenidos, muy acordes al código del mensaje que se ha impuesto en la red social, también están relacionados con los entrenamientos $\mathrm{y}$, en menor medida, con aquellas publicaciones que se centran en un solo jugador.

En este mismo sentido, los hashtagchallenges, que procuran mayor participación e implicación por parte de los usuarios con los contenidos, no destacan como una prioridad a la hora de publicar, aunque la mayoría de las propuestas se procuran hacer en tono entretenido.

Cuando los equipos acuden a contenidos anecdóticos, lo hacen sobre todo para exaltar la figura de un jugador en particular, aunque también para enaltecer la identidad y tradición del club.

Por último, los contenidos como parte de una estrategia de comunicación corporativa son evidentemente minoritarios, pero cuando hay 
evidencia de ellos, en su mayoría están relacionados con una necesidad de reforzar la imagen e identidad de la institución. Muy poco se hace uso del recurso para aludir a eventos, competiciones destacadas o alusiones específicas a grandes estrellas con vinculación directa con el equipo, que también fortalecen esa imagen.

\section{Discusión}

La pandemia mundial provocada por la COVID-19 y las medidas derivadas de la situación de emergencia sanitaria, como el confinamiento de la población en sus hogares, dieron lugar a un incremento en el tiempo dedicado al consumo de redes sociales, así como a un aumento en las descargas de algunas de ellas, como es el caso de TikTok. La red social se convierte en tendencia en 2020 y los clubes de fútbol no son indiferentes a este fenómeno.

\section{Evolución narrativa de los clubes de fútbol en TikTok}

Los equipos de la Liga Santander ven en TikTok un nuevo canal de comunicación, especialmente durante el segundo trimestre de 2020, que es cuando un mayor número de clubes crean una cuenta oficial en esta red social (Figura 1). De esta manera, se verifica la primera hipótesis de la presente investigación.

Cabe destacar que con anterioridad al año 2020 clubes como el Cádiz C.F., el Real Madrid y el Atlético de Madrid ya disponían de un perfil en
TikTok, pero con una narrativa más alejada de la que caracteriza hoy a la red social. No obstante, este es un detalle importante porque, aunque TikTok aún no había escalado como fenómeno digital global, reportaba un aumento importante de uso en varios países, lo que demuestra que los clubes de fútbol tienen una intención cada vez mayor de trabajar su marca a través de tribunas alternativas y novedosas.

En el caso concreto del Cádiz C.F., las primeras publicaciones son vídeos tomados del canal de televisión del equipo de fútbol, los cuales se publican en TikTok con el formato horizontal, con audio e incluso el logotipo en pantalla del vídeo original.

Un caso similar sucede con el Granada C.F. Esto se puede atribuir al interés de los clubes por incorporarse a las nuevas plataformas, aún sin tener muy bien definidas las estrategias de comunicación (Sotelo, 2012), aunque posteriormente, se aprecia una adaptación de las publicaciones al formato propio de la narrativa de TikTok, con contenidos concebidos expresamente para esta red social, con un formato de video vertical y el empleo de filtros, efectos y pistas de audio.

Se ha tratado de un proceso muy rápido ya que en cuestión de menos de un año todos los clubes de la Liga Santander han abierto una cuenta en TikTok y la mayoría publica de forma frecuente. De esta manera, ha habido una adaptación en caliente a nuevas formas narrativas alejadas del vasto trabajo adelantado en redes sociales con mayor trayectoria como Instagram, Twitter y Facebook. Así, en líneas generales, se ha producido una adaptación de los clubes a la narrativa propia de la red social, que, en

Global Media Journal México, 18(35), 32-54, julio - diciembre 2021. 
un primer momento, les podía resultar ajena (Sidorenko et al., 2020; Vásquez et al., 2020).

Sin embargo, como parte de este proceso también se aprecia un cansancio o dificultad por acompañar la velocidad de los acontecimientos. Así, se produce un fenómeno común en varios clubes con respecto al descenso en la frecuencia de publicación desde el inicio de la cuenta. Es el caso del Osasuna, el Villarreal C.F., el Levante y el Sevilla F.C. El primero, por ejemplo, una vez finaliza la competición de Liga 2019-2020 el 19 de julio de 2020, su frecuencia de publicación decae considerablemente, hasta encontrar el último contenido publicado el 9 de febrero de 2021.

Otro ejemplo quizás más llamativo es el del SD Huesca, que, si bien empezó su actividad en TikTok a partir del segundo trimestre de 2020, no ha publicado más contenidos desde diciembre del mismo año. Y es que, pese a que la observación de este estudio se ha fijado hasta el 31 de diciembre 2020 para evaluar la actividad de los clubes en el “año de la explosión de TikTok", observaciones posteriores a dicha periodización han permitido obtener la evidencia expuesta hasta el primer cuatrimestre de 2021.

En la mencionada evolución que se encuentra en varios de los perfiles analizados hacia una narrativa más propia o nativa de la red social, se observan diversos contenidos que coinciden en la forma y en empleo de un tono más informal y divertido.

Es el caso de los videos en los que los jugadores van cambiando de uniformes dando un toque al pecho y al ritmo de la música, un recurso utilizado también para obtener mayor engagement por parte de los seguidores (convertido en hashtagchallenge como el caso del SD Huesca, véase https://bit.ly/3y8n8BB y https://bit.ly/3hrRDMS) o inclusive con un intento de impacto publicitario para intentar comercializar un mayor número de camisetas entre los aficionados.

Destaca también en varios perfiles la presencia de la mascota de los clubes. De hecho, muchos de los contenidos entretenidos del Atlético de Madrid, el Valencia C.F., el Valladolid y la Real Sociedad relacionados con eventos y competiciones implican la participación de la mascota.

El contenido de los clubes de fútbol de La Liga Santander en TikTok está construido mayoritariamente sobre una narrativa en clave publicitaria o de carácter anecdótico con especial énfasis en la imagen del club.

De esta manera, queda en evidencia que la segunda hipótesis, la que hacía referencia al contenido que los clubes difundían en TikTok y que podría construirse en torno a una narrativa en clave publicitaria o de carácter anecdótico, no es posible confirmarla ya que resultan escasos en proporción con otras categorías encontradas, los contenidos de carácter publicitario e incluso anecdótico (Tabla 2).

Prevalecen los contenidos de carácter más informativo sobre todo que aluden a los entrenamientos o la preparación de los diversos encuentros y competiciones. Se trata de videos que carecen de música, filtros y efectos en muchas ocasiones, aunque el formato es vertical. Sin embargo, el Atlético de Madrid, por ejemplo, acompaña con mayor frecuencia sus videos de 
entrenamientos con la música tendencia del momento, la cual es una variable importante para procurar mayor visibilidad de manera orgánica por parte del algoritmo de TikTok frente a la comunidad de usuarios de la plataforma.

Al hilo de la idea de "narrativa TikTok", en el caso particular de los ya referenciados retos o challenges, vale la pena aludir a casos específicos como el del Athletic de Bilbao con el hashtag \#diadeloszurdos, para proponer el reto de hacer el mayor número de toques de balón con la pierna y el pie izquierdo. En el caso del F.C. Barcelona, algunos retos destacados se pueden identificar como \#impossibletricks, \#guesswho, \#10toqueschallenge o \#laligachek. Sin embargo, estos dos últimos son transversales a otros equipos como el Athletic de Bilbao, el Elche y el Osasuna, sin mencionar casos como \#juegodeelectricidad, \#imitaelemoji, \#TikStop, \#carltondance o el \#onananachallenge (véase https://bit.ly/3kMOia1

$\mathrm{y}$
https://bit.ly/2JqpTcO) que son promovidos por las audiencias y en los que los equipos buscan participar para ser parte de la tendencia.

En todo caso, se trata de iniciativas que van en consonancia con la dinámica y estética impuesta en el seno de la red social y que persiguen mayor acercamiento e implicación con los seguidores.

\section{TikTok como elemento para la comunicación corporativa de los clubes de fútbol}

Al margen de todo ello, también fue posible encontrar un vasto catálogo de videos que acentúan la comunicación corporativa de estos equipos, con enfoque en los entrenamientos (véase https://bit.ly/3jL9Tyu o https://bit.ly/35U10Qy), en celebraciones (véase https://bit.ly/2HSihyM), como spots informativos para diversos segmentos de público (véase https://bit.ly/3kJMkHK), sobre eventos de pretemporada (véase https://bit.ly/2TG1uBR), la vuelta de La Liga (19-20) (véase https://bit.ly/3mHR7do), publicidad de videojuegos (véase https://bit.ly/3kOtzTi), publicidad de marcas de coches (véase https://bit.ly/2HQqTGg) o diseñadores de ropa (véase https://bit.ly/3oLRi9f) y sinergias con artistas (véase https://bit.ly/37YT7d2, https://bit.ly/385RpX4 o https://bit.ly/3mJxO3o).

En el caso concreto del Atlético de Madrid, el equipo plasmó a través de TikTok de manera pormenorizada su pretemporada en Estados Unidos y México, documentando todos los eventos, competiciones y actividades deportivas y extradeportivas en las que fueron participando. Por su parte, el F.C. Barcelona visualizó en su perfil los viajes del equipo a las diferentes competiciones europeas, así como la asistencia a eventos extradeportivos, como la ópera en la Scala de Milán.

Valdría destacar también al Cádiz C.F, el cual publicó un vídeo con un barco acompañado del texto "Elcano viaja por el mundo con nuestra bandera" o el asistente de voz Alexa alabando al club al ser preguntado por él.

Es posible encontrar numerosas publicaciones recuperando jugadas míticas de los clubes y homenajes a jugadores reconocidos que pasaron por el club, como puede ser el portero Iker Casillas en el Real Madrid, Aduritz en el Athletic de 
Bilbao, Mario Kempes en el Valencia y Ronaldinho o Ronald Koeman en el Barcelona, porque aparte de los entrenamientos o el enfoque en jugadores particulares (leyendas o nuevos fichajes) corresponde a otro recurso muy utilizado a fin de reforzar la marca del club (Figura 2).

Ha sido posible observar cómo los propios jugadores se constituyen por un lado como marcas en sí mismos dentro de los perfiles de los clubes, adquiriendo el rol de "futbolistas sociales" (Marini, 2018), y, por otra parte, como ya se ha puntualizado, como elementos que refuerzan la imagen de marca de estas instituciones (Miranda Peña, 2021; Omar \& Dequan, 2020).

En los contenidos estudiados en TikTok, a los jugadores se les presenta con videos entretenidos en muchos casos, se les felicita los cumpleaños y se les realiza seguimientos personalizados e individualizados en las jugadas de los partidos y en los entrenamientos. Asimismo, en el caso particular del F.C. Barcelona, cuando un jugador abandona el club, se le muestra en un vídeo con su familia vaciando su propia taquilla.

En otros contenidos también se busca enaltecer a los jugadores más destacados de una jornada. Es el caso de Antoine Griezmann, que es protagonista de una considerable cantidad de videos en el perfil del F.C. Barcelona, tanto en su faceta de jugador en los partidos o en los entrenamientos, como con su familia, todos acompañados de su propio hashtag, \#antoinegriezmann. Un caso que también se aprecia fácilmente con Lionel Messi. En la plataforma se le muestra en diferentes momentos, en el campo y fuera de él, como un héroe deportivo recogiendo el trofeo del Balón de Oro y un jugador imbatible en el terreno de juego, reproduciendo sus jugadas con su propio hashtag, \#Messi.

Quizás lo más llamativo y que corresponde a esa importancia que cobran los audios como variables de cara al algoritmo de TikTok, es el hecho de que Messi cuenta con música propia, lo que denota la relevancia de su figura para el club y el impacto/alcance con respecto a la comunidad de usuarios que el F. C. Barcelona ha deseado conseguir a través de esta red social (véase https://vm.tiktok.com/ZMeg93Ykm/).

Tras los argumentos esgrimidos hasta este punto, así como en referencia a los datos recogidos en la Figura 2 y la Tabla 2, es posible afirmar que la comunicación de los clubes de fútbol de la primera división española en esta red social está ligada en gran medida a la propia comunicación corporativa del club, por lo que no se cumple tampoco la tercera hipótesis. Es decir, el trabajo que este tipo de instituciones adelanta a través de tan particular vía, si bien no tiene una intención expresa de reforzar o incrementar el alcance de su identidad corporativa (Tabla 2), con TikTok han conseguido sumar una nueva tribuna con grandes posibilidades de llegar a segmentos de usuarios más jóvenes que hoy parecieran tener una mayor desconexión con los deportes convencionales como ya ha advertido Aguilar (2021).

Sin embargo, como se ha señalado en líneas anteriores, tanto los propios clubes en sí mismos, como a través de sus jugadores, constituyen a través de TikTok un reclamo publicitario que muchas veces se difumina con la comunicación corporativa 
propiamente señalada. Un ejemplo de ello lo constituye la empresa canadiense de videojuegos EA Sports, responsable de los populares títulos "FIFA", la cual realiza colaboraciones con diversos clubes de La Liga Santander con el fin de promocionar su producto, y, en el caso particular del Atlético de Madrid, destaca una implicación-colaboración aún mayor. Algunos ejemplos que bien valdrían destacarse son: https://bit.ly/3n59evb, https://bit.ly/3auASMV y https://bit.ly/2QN0E7W, donde jugadores y entrenadores no solo destacan la importancia del producto (videoconsola y videojuego) sino que destacan su propia imagen como puente de estos productos.

Ello invita a la reflexión sobre cómo marcas y empresas ven en clubes como este en particular, una tribuna publicitaria con alto impacto y alcance, reforzándose así la imagen de ambas instancias.

Por su parte, es el caso particular del Valencia C.F., el Valladolid y el Sevilla F.C., que han dedicado contenidos donde los propios jugadores son convertidos en avatares del videojuego Among Us. En resumen, es posible ver una sinergia por parte de estas instituciones deportivas con marcas y empresas de otros ámbitos, así como un empeño por incursionar de manera indirecta o muy directa en territorios que están obteniendo una importante atención por parte de los públicos emergentes y más digitales.

\section{Iniciativas solidarias y buenas prácticas a través de TikTok}

Por motivos similares, la presencia en los perfiles de TikTok de iniciativas solidarias es un importante elemento corporativo para la imagen del club. Valgan como ejemplo las publicaciones realizadas por el Athletic de Bilbao apoyando diferentes causas sociales con los hashtags \#blacklivesmatter $\mathrm{y}$ \#Diadelorgullo. En algunas publicaciones es posible ver las manos de jugadores con pulseras en las que se puede leer STOP Racism y en la segunda se observa el estadio iluminado con los colores del arco iris.

En la misma línea, durante los meses de confinamiento, se encuentran varias publicaciones con los jugadores entrenando en sus hogares y mandando mensajes de ánimo, así como realizándose test en la vuelta a los entrenamientos. Algo que fue común ver por parte de otras franquicias deportivas, como el caso de la Asociación Nacional de Baloncesto de Estados Unidos (NBA, por sus siglas en inglés).

No obstante, un detalle que no pasa desapercibido es el hecho de no darle prácticamente visibilidad a la hinchada, teniendo en cuenta que se trata de un deporte donde la afición es un elemento primordial (por aquello del "jugador $\mathrm{N}^{\mathrm{o}} 12$ ”). Son escasos los contenidos que aluden expresamente a los fanáticos, más aún en un año tan particular donde los estadios se han visto imposibilitados de permitir el acceso a los eventos deportivos como consecuencia de la COVID-19.

Un detalle relevante en el marco de la revisión de casos ha sido la observación del incremento de la presencia del fútbol femenino en la mayor parte de las cuentas de TikTok de los clubes a lo largo de 2020, aunque en la oferta de contenidos predominan los equipos masculinos. Sin embargo, 
casos como el SD Huesca, Real Sociedad o el Valencia han creado ya perfiles diferenciados.

Hay que poner en valor y destacar el hecho de cada vez hay mayor presencia en los perfiles de los clubes de fútbol español, de contenido ideado y creado para la propia red social, es decir, que corresponden a una estrategia de comunicación diferenciada con respecto a otras plataformas digitales. Corresponde al Cádiz C.F. el mejor ejemplo para evidenciar esta evolución, donde las últimas publicaciones incorporan recursos de edición audiovisual (transiciones, diálogos, animaciones y música) de la propia red social. Asimismo, equipos como el Valencia C.F., el Celta y el Villarreal, han procurado con mayor frecuencia diferentes challenges, coreografías y empleo de recursos narrativos propios de TikTok.

Por último, en cuanto a si el desarrollo de contenidos en TikTok por parte de los clubes de fútbol de La Liga Santander es aún incipiente y experimental y que, en consecuencia, hay una escasa producción multimedia por parte de los emisores ya referenciados, se puede afirmar rotundamente que no, aunque también es necesario precisar que hay una importante desigualdad en la determinación y capacidad de producción por parte de los diferentes perfiles. Hay clubes como el Real Madrid, el Barcelona o el Atlético de Madrid que ostentan una ingente producción multimedia en TikTok, mientras que el Getafe ha sido irregular en este proceso y el Cádiz C.F. por su parte desde diciembre de 2020 no ha vuelto a publicar, por hacer referenciar algunos casos en concreto.
De esta manera se puede sentenciar que no se pudo confirmar tampoco la cuarta hipótesis en conjunto, pero quizás si se estudian a profundidad la actividad de manera independiente por club se logre su comprobación (Tabla 1).

\section{Conclusiones}

El hecho de que TikTok se haya convertido en tendencia digital en un corto período de tiempo y en un contexto global tan particular como lo ha sido la pandemia por COVID-19, hizo que muchas empresas, marcas e instituciones sintieran la necesidad de acudir en primera instancia a esta tribuna comunicativa como una nueva alternativa para incrementar el alcance de la imagen y el mensaje, en ocasiones sin tener muy claro en qué consistía. En este sentido, el particular código del mensaje impuesto por la comunidad de usuarios puede haber sido algo determinante en la experimentación y una aparente falta de criterios concretos iniciales para hacerlo (Sidorenko et al., 2021).

En el caso particular de los clubes de la Liga Santander, ha sido posible observar que aproximadamente la mitad (en lo que corresponde a actores de la temporada 20/21) ya partían de una situación aventajada al incursionar en la red social desde 2019, previendo el ascenso que tenía sobre todo en segmentos de audiencia muy joven, aunque en algunos casos los contenidos publicados no fueran 
creados específicamente para TikTok, sino recuperados de otros canales de difusión.

Sin embargo, este fenómeno global respecto a TikTok que se aprecia a partir de 2020 obligó al resto de equipos a sumarse a la tendencia de manera apresurada, sin medir bien la manera de hacerlo, dejando en evidencia una falta de estrategia a la hora de comunicar. También es necesario destacar el hecho de que el Real Betis Balompié disponía de un perfil verificado en TikTok sin haber publicado ningún contenido hasta el primer cuatrimestre de 2021.

Como se ha puntualizado, en varias ocasiones los primeros contenidos de los clubes no han estado precisamente adaptados a la narrativa vertical de TikTok, siendo estas publicaciones originales de televisión o de plataformas como YouTube o Instagram. Luego sí es cierto que, una vez comprendida la dinámica sobre los efectos, audios y challenges, incrementaron su actividad y con ello han construido una relación más cercana e interactiva con sus seguidores.

En la mayoría de los casos revisados se deja en evidencia una intención expresa por enaltecer la imagen e identidad de la institución deportiva. Sin embargo, prevalece una desigualdad muy importante en la determinación de producción multimedia entre los diferentes equipos estudiados.

La hinchada es muy poco o nada visibilizada en las publicaciones de todos los equipos estudiados. Es comprensible que se ha tratado de una temporada atípica sin asistencia de público por las restricciones satinarías en el marco de la pandemia por COVID19. No hay contenidos que valga la pena resaltar que enaltezcan a los seguidores y que les representen pese a la distancia impuesta por la coyuntura.
No obstante, sí que todos estos esfuerzos de abrir y dotar de contenido y nuevas vías de comunicación a este tipo de instituciones deportivas están enfocados no solo en reforzar la fidelidad de seguidores y fanáticos, sino especialmente en captar nuevos aficionados pertenecientes a los segmentos de audiencia más jóvenes, los cuales quizás están más desconectados de los deportes tradicionales frente a otras opciones como los eSports y videojuegos.

En este punto, una explicación remite a la búsqueda de un engagement con los propios aficionados a través de los elementos propios y de identidad de los clubes y con los que la afición se identifica, como el estadio, los jugadores o el escudo del club, abriendo así una nueva ventana en forma de canal de comunicación para los seguidores de los equipos, a través de la que también pueden ver jugadas relevantes de los partidos y momentos destacados de los entrenamientos.

De hecho, los equipos se apoyan mucho en los contenidos publicados en TikTok en la imagen de los jugadores y de los entrenadores (fichajes y leyendas) para reforzar la identidad de club. Mientras tanto, los jugadores también ven fortalecida su marca personal a través de hashtags e inclusive audios.

Quedará para futuras investigaciones llevar a cabo comparaciones de la actividad digital de estos clubes en TikTok con respecto al resto de redes sociales donde tienen perfiles activos. Asimismo, sería pertinente extender este tipo de observación al ámbito del fútbol europeo y latinoamericano, con el fin de entender mejor el funcionamiento de TikTok como fenómeno global enfocado en la comunicación efímera y sobre estimulada visualmente, pero con un componente relevante también para la comunicación corporativa e imagen de marca 


\section{Referencias bibliográficas}

Aguilar, L. (2021, April 21). Ligas LoL: ¿Acabará Twitch con las retransmisiones deportivas tradicionales?. Yorokobu. https://bit.ly/3ksCYBZ

Almansa Martínez, A, \& Castillo Esparcia, A. (2014). Comunicación institucional en España. Estudio del uso que los diputados españoles hacen de las TIC en sus relaciones con la ciudadanía. Revista Chasqui, 126, 2230. https://bit.ly/3oiXGoT

Cano Tenorio, R. (2017). Técnicas de marketing de los clubes de fútbol de élite en las redes sociales. Retos, 13(7), 43-58. http://dx.doi.org/10.17163/ret.n13.2017.03

Castillo Esparcia, A., Fernández Torres, M. J., \& Castillero Ostío, E. (2016). Fútbol y redes sociales. Análisis de la gestión de relaciones públicas 2.0 por los clubes de fútbol. Estudios sobre el Mensaje Periodístico. 22(1), 239-254. https://doi.org/10.5209/rev_ESMP.2016.v22.n1.52592

Chan, J. (2021, 4 de mayo). Top apps worldwide for April 2021 by downloads. Sensor Tower. https://bit.ly/3euF6Xb

Cobo Romaní C., \& Pardo Kuklinski, H. (2007). Planeta web 2.0 inteligencia colectiva o medios fast food. Facultad Latinoamericana de Ciencias Sociales.

Ditrendia (2020). Móviles en España y en el Mundo 2020. Ditrendia. https://bit.ly/3iY6iwV

Ginesta, X. (2011). Football and the global entertainment business. Clubs as entertainment multinational corporations. Communication \& Society, 24(1), 141-166. https://bit.ly/3Hf3eJW

Gozàlvez, V. (2012). Ciudadanía mediática. Una mirada educativa. Dykinson.

IAB (2021). Estudio de redes sociales 2021. https://bit.ly/3uwjqQd

Kantar (2020, July 15). TikTok is the brand to watch: BrandZ global top 100 2020. http://bit.ly/3a19iXM

López Martínez, R., \& Fernández Fernández, J. (2015). Responsabilidad social corporativa y buen gobierno en los clubes de fútbol españoles. Universia Business Review, 2, 38-53. https://bit.ly/3n6cUyo

Li, X., Xiaohui, Y., \& Zhengwu, Z. (2019). Research on the causes of the «TikTok» app becoming popular and the existing problems. Journal of Advanced Management Science, 7(2), 59-63. https://doi.org/10.18178/joams.7.2.59-63

Marini, E. (2018). Las redes sociales como estrategia comunicativa en la espectacularización del deporte: el caso del fútbol de masas en España, Italia e Inglaterra, [Tesis doctoral no publicada]. Facultad de Ciencias de la Información, Universidad Complutense de Madrid.

Meneses, G. A., \& Ávalos González, J. M. (2013): La investigación del futbol y sus nexos con los estudios de comunicación: Aproximaciones y ejemplos. Comunicación y sociedad, 20, 33-64. https://bit.ly/2YD5Hwj

Miranda Peña, A. C. (2021). Las redes sociales un área de oportunidad en el fútbol. Summa Humanitatis, 11(2), 1-15. https://bit.ly/3oqrIaC

Global Media Journal México, 18(35), 32-54, julio - diciembre 2021. 
Omar, B. \& Dequan, W. (2020). Watch, share or create: the influence of personality traits and user motivation on TikTok mobile video usage. International Journal of Interactive Mobile Technologies, 14(4), 121-137 https://doi.org/10.3991/ijim.v14i04.12429

Permana, I. P. H. \& Meinarni, N. P. S. (2021). Ratio analysis on Tiktok (social media) for qualitative research using explorative methods. Jurnal Ekonomi dan Bisnis Jagaditha, 8(1), 30-38. https://doi.org/10.22225/jj.8.1.2944.30-38

Rapkin, M. (2017, November 1). The social media platform that has Gen Z obsessed. Wall Street Journal Magazine. https://on.wsj.com/2YVWhZj

Rheingold, H. (2002). Smart mobs: the next social revolution. Perseus Books Group.

Rojas-Torrijos, J. L. (2012). La futbolización de la información deportiva. Un estudio de casos de cuatro diarios deportivos europeos. Comunicação \& Cultura, 13, 77-95. https://bit.ly/3wDTnZ1

Ruihley, B. J., \& Li, B. (2020). Sport and the coronavirus crisis special issue: an introduction. International Journal of Sport Communication, 13, 289-293. https://doi.org/10.1123/ijsc.2020-0254

Sehl, K. (2020, May 7). 20 important TikTok stats marketers need to know in 2020. Hoosuite Blog. https://bit.ly/31NnOoJ

Shuai, Y., Yuzhen, Z., \& Yifang, M. (2019). Analysis of the reasons and development of short video application - Taking Tik Tok as an example. 9th International Conference on Information and Social Science (ICISS 2019). Francis Academic Press, 340-343. https://doi.org/10.25236/iciss.2019.062

Sotelo González, J. (2012). Deporte y social media: el caso de la primera división del fútbol español. Historia y Comunicación Social, 17, 217-230. https://doi.org/10.5209/rev_HICS.2012.v17.40607

Soriano, F. (2009). La pilota no entra per atzar. Ara llibres.

Sidorenko Bautista, P., Herranz de la Casa, J. M., \& Cantero de Julián, J. I. (2020). Use of new narratives for COVID-19 reporting: from $360^{\circ}$ videos to ephemeral TikTok videos in online media. Tripodos, $47(1)$, 105-122. https://doi.org/10.51698/tripodos.2020.47p105-122

Sidorenko Bautista, P., Herranz de la Casa, J. M., \& Moya Ruiz, A. S. (2021) Análisis de la comunicación de empresas europeas y norteamericanas en TikTok. aDResearch ESIC. 25(25), 106-123. https://doi.org/10.7263/adresic-025-06

Tejedor, S., Cervia, L., \& Vecino, C. (2020). El fútbol en las redes sociales: Análisis de la presencia en Facebook de los principales equipos de Europa. Ibero-American Journal of Exercise, Sports and Psychology, 15(3), 150-156. https://bit.ly/30ax2pY

Terol Bolinches, R. (2016). Radio 3.0 en el entorno municipal: valores, herramientas y recursos. El caso de la emisora municipal Llosa FM, [Tesis doctoral no publicada]. Universitat Politècnica de València. https://oi.org/10.4995/Thesis/10251/67930

Global Media Journal México, 18(35), 32-54, julio - diciembre 2021. 
Vázquez-Herrero, J., Negreira-Rey, M. C., \& López-García, X. (2020). Let's dance the news! How the news media are adapting to the logic of TikTok. Journalism, 1-19. https://doi.org/10.1177/1464884920969092

Van den Bremer, A. J. M. \& Siebelink, R. (2020). Critical Thinking on TikTok. Universidad de Utrecht, [Bachelor thesis]. Disponible en https://dspace.library.uu.nl/handle/1874/400672

Yu-Liang, F. Y. L., Chun-Chin, C., \& Shu-Ming, W. (2019). Evaluation of charm factors of short video user experience using FAHP - a case study of TikTok app. IOP Conference Series: Materials Science and Engineering, 688, 1-5. https://doi.org/10.1088/1757-899X/688/5/055068

\section{Notas}

\footnotetext{
' Por convención mundial en el ámbito de la comunicación, sobre todo digital, se hace referencia como Boomers a aquellos usuarios que en la actualidad se encuentran en un rango de edad entre los 58 y 65 años, 'Generación X' entre 39-57 años, Millennials entre 25 y 38 años y 'Generación Z' o Centennials entre 17 y 24 años aproximadamente. Para más información véase el estudio pormenorizado sobre hábitos de consumo digital presentado en 2020 por Visual Capitalist (https://www.visualcapitalist.com/media-consumption-covid-19/).
} 